\title{
Coupled Common Fixed Point Theorems in Partially Ordered $G$-metric Spaces for Nonlinear Contractions
}

\author{
Manish Jain, Calogero Vetro, \\ NeEtu Gupta And Sanjay Kumar
}

\begin{abstract}
The aim of this paper is to prove coupled coincidence and coupled common fixed point theorems for a mixed $g$-monotone mapping satisfying nonlinear contractive conditions in the setting of partially ordered $G$-metric spaces. Present theorems are true generalizations of the recent results of Choudhury and Maity [Math Comput. Modelling 54 (2011), 73-79], and Luong and Thuan [Math. Comput. Modelling 55 (2012), 1601-1609].
\end{abstract}

\section{Introduction And Preliminaries}

In [12], Mustafa together with Sims introduced the generalized structure of metric spaces, called $G$-metric spaces. Afterwards, numerous fixed point theorems in this generalized structure were proved by different authors. Works noted in $[1,6,9,11,13,14,17,21]$ are some examples in this direction. Bhaskar and Lakshmikantham [3] introduced the notion of coupled fixed point and proved some coupled fixed point theorems for a mapping satisfying mixed monotone property in partially ordered metric spaces. As an application, they discussed the existence and uniqueness of solution for a periodic boundary value problem. Lakshmikantham and Ćirić [8] extended the notion of mixed monotone property to mixed $g$-monotone property and generalized the results of Bhaskar and Lakshmikantham [3] by establishing the existence of coupled coincidence point results using a pair of commutative mappings. These results have been extended and generalized by several authors. References $[7,15,16]$ are some examples of these works. Now-a-days authors have keen interest in proving fixed point theorems in partially ordered metric spaces subjected to nonlinear contractive conditions, see $[2,4,10,18-20]$. Our paper deals with the establishment of some coupled coincidence and

2010 Mathematics Subject Classification. Primary: 47H10; Secondary: 54H25.

Key words and phrases. Partially ordered set, $G$-metric space, coupled coincidence point, coupled common fixed point, mixed monotone mappings. 
coupled common fixed point results for a mixed $g$-monotone mapping satisfying nonlinear contractive conditions in partially ordered $G$-metric spaces. Our results generalize the recent results of Choudhury and Maity [5] and Luong and Thuan [9]. We give also an example to illustrate our results. We now recall some definitions and properties in $G$-metric spaces (see [12]).

Definition 1.1. Let $X$ be a nonempty set. Suppose that $G: X \times X \times X \rightarrow$ $[0,+\infty)$ is a function satisfying the following conditions:

(G1) $G(x, y, z)=0$ if and only if $x=y=z$;

(G2) $0<G(x, x, y)$ for all $x, y \in X$ with $x \neq y$;

(G3) $G(x, x, y) \leq G(x, y, z)$ for all $x, y, z \in X$ with $y \neq z$;

(G4) $G(x, y, z)=G(x, z, y)=G(y, z, x)=\ldots$ (symmetry in all three variables);

(G5) $G(x, y, z) \leq G(x, a, a)+G(a, y, z)$ for all $x, y, z, a \in X$ (rectangle inequality).

Then $G$ is called a $G$-metric on $X$ and $(X, G)$ is called a $G$-metric space.

Definition 1.2. Let $(X, G)$ be a $G$-metric space. We say that $\left\{x_{n}\right\}$ is:

(i) a $G$-Cauchy sequence if, for any $\varepsilon>0$, there is an $N \in \mathbb{N}$ (the set of all positive integers) such that for all $n, m, l \geq N, G\left(x_{n}, x_{m}, x_{l}\right)<\varepsilon$;

(ii) a $G$-convergent sequence to $x \in X$ if, for any $\varepsilon>0$, there is $N \in \mathbb{N}$ such that for all $n, m \geq N, G\left(x, x_{n}, x_{m}\right)<\varepsilon$.

A $G$-metric space $(X, G)$ is said to be complete if every $G$-Cauchy sequence in $X$ is $G$-convergent in $X$.

Proposition 1.1. Let $(X, G)$ be a $G$-metric space. The following are equivalent:

(i) $\left\{x_{n}\right\}$ is G-convergent to $x$;

(ii) $G\left(x_{n}, x_{n}, x\right) \rightarrow 0$ as $n \rightarrow+\infty$;

(iii) $G\left(x_{n}, x, x\right) \rightarrow 0$ as $n \rightarrow+\infty$;

(iv) $G\left(x_{n}, x_{m}, x\right) \rightarrow 0$ as $n, m \rightarrow+\infty$.

Proposition 1.2. Let $(X, G)$ be a $G$-metric space. Then the following are equivalent:

(i) the sequence $\left\{x_{n}\right\}$ is G-Cauchy;

(ii) $G\left(x_{n}, x_{m}, x_{m}\right) \rightarrow 0$ as $n, m \rightarrow+\infty$.

Proposition 1.3. Let $(X, G)$ be a $G$-metric space. A mapping $g: X \rightarrow X$ is $G$-continuous at $x \in X$ if and only if it is $G$-sequentially continuous at $x$, that is, whenever $\left\{x_{n}\right\}$ is $G$-convergent to $x,\left\{g\left(x_{n}\right)\right\}$ is $G$-convergent to $g(x)$.

Proposition 1.4. Let $(X, G)$ be a $G$-metric space. Then the function $G(x, y, z)$ is jointly continuous in all three of its variables. 
Definition $1.3([5])$. Let $(X, G)$ be a $G$-metric space. A mapping $F: X \times$ $X \rightarrow X$ is said to be continuous if for any two $G$-convergent sequences $\left\{x_{n}\right\}$ and $\left\{y_{n}\right\}$ converging to $x$ and $y$ respectively, $\left\{F\left(x_{n}, y_{n}\right)\right\}$ is $G$-convergent to $F(x, y)$.

An interesting observation is that any $G$-metric space $(X, G)$ induces a metric $d_{G}$ on $X$ given by

$$
d_{G}(x, y)=G(x, y, y)+G(y, x, x), \text { for all } x, y \in X .
$$

Moreover, $(X, G)$ is $G$-complete if and only if $\left(X, d_{G}\right)$ is complete.

Now, we recall some definitions introduced in $[3,8]$. Let $(X, \preceq)$ be a partially ordered set and $g: X \rightarrow X$ be a mapping. The mapping $g$ is said to be non-decreasing if for all $x, y \in X, x \preceq y$ implies $g(x) \preceq g(y)$. Similarly, $g$ is said to be non-increasing, if for all $x, y \in X, x \preceq y$ implies $g(x) \succeq g(y)$.

Bhaskar and Lakshmikantham [3] introduced the following notions of mixed monotone mapping and coupled fixed point.

Definition 1.4. Let $(X, \preceq)$ be a partially ordered set and $F: X \times X \rightarrow$ $X$. The mapping $F$ is said to have the mixed monotone property if $F$ is monotone non-decreasing in its first argument and is monotone nonincreasing in its second argument, that is, for all $x_{1}, x_{2} \in X, x_{1} \preceq x_{2}$ implies $F\left(x_{1}, y\right) \preceq F\left(x_{2}, y\right)$, for any $y \in X$ and for all $y_{1}, y_{2} \in X, y_{1} \preceq y_{2}$ implies $F\left(x, y_{1}\right) \succeq F\left(x, y_{2}\right)$, for any $x \in X$.

The concept of the mixed monotone property was generalized by Lakshmikantham and Ćirić [8] as follows.

Definition $1.5([8])$. Let $(X, \preceq)$ be a partially ordered set and $F: X \times$ $X \rightarrow X$ and $g: X \rightarrow X$. The mapping $F$ is said to have the mixed $g$ monotone property if $F$ is monotone $g$-non-decreasing in its first argument and is monotone $g$-non-increasing in its second argument, that is, for all $x_{1}, x_{2} \in X, g\left(x_{1}\right) \preceq g\left(x_{2}\right)$ implies $F\left(x_{1}, y\right) \preceq F\left(x_{2}, y\right)$, for any $y \in X$ and for all $y_{1}, y_{2} \in X, g\left(y_{1}\right) \preceq g\left(y_{2}\right)$ implies $F\left(x, y_{1}\right) \succeq F\left(x, y_{2}\right)$, for any $x \in X$.

Clearly, if $g$ is the identity mapping, then Definition 1.5 reduces to Definition 1.4.

Definition 1.6. An element $(x, y) \in X \times X$ is called a coupled fixed point of the mapping $F: X \times X \rightarrow X$ if $F(x, y)=x$ and $F(y, x)=y$.

Definition 1.7. An element $(x, y) \in X \times X$ is called a coupled coincidence point of the mappings $F: X \times X \rightarrow X$ and $g: X \rightarrow X$ if $F(x, y)=g(x)$ and $F(y, x)=g(y)$.

Definition 1.8. Let $F: X \times X \rightarrow X$ and $g: X \rightarrow X$. Then, $F$ and $g$ are said to be commutative if $g(F(x, y))=F(g(x), g(y))$ for all $x, y \in X$.

Definition 1.9 ([10]). Let $\Phi$ denote all functions $\phi:[0,+\infty) \rightarrow[0,+\infty)$ such that 
(i) $\phi$ is continuous and increasing;

(ii) $\phi(t)=0$ iff $t=0$;

(iii) $\phi(t+s) \leq \phi(t)+\phi(s)$, for all $t, s \in[0,+\infty)$.

Definition $1.10([9])$. Let $\Theta$ denote all functions $\theta:[0,+\infty) \times[0,+\infty) \rightarrow$ $[0,+\infty)$ such that $\lim _{\left(t_{1}, t_{2}\right) \rightarrow\left(r_{1}, r_{2}\right)} \theta\left(t_{1}, t_{2}\right)>0$ for all $\left(r_{1}, r_{2}\right) \in[0,+\infty) \times$ $[0,+\infty)$ with $r_{1}+r_{2}>0$.

Let $(X, \preceq)$ be a partially ordered set, $F: X \times X \rightarrow X$ be a mapping having the mixed monotone property and suppose there exists a $G$-metric such that $(X, G)$ is a $G$-metric space. Choudhury and Maity [5] established some fixed point results for the mapping $F$ under the following contractive condition

$$
G(F(x, y), F(u, v), F(w, z)) \leq \frac{k}{2}(G(x, u, w)+G(y, v, z))
$$

for $w \preceq u \preceq x$ and $y \preceq v \preceq z$, where $k \in[0,1)$.

Recently, Luong and Thuan [9] generalized the results of Choudhury and Maity [5] by proving some coupled fixed point theorems in partially ordered $G$-metric spaces under a nonlinear contractive condition of the form

$$
\begin{aligned}
& G(F(x, y), F(u, v), F(w, z)) \leq \\
& \frac{G(x, u, w)+G(y, v, z)}{2}-\theta(G(x, u, w), G(y, v, z))
\end{aligned}
$$

for $w \preceq u \preceq x$ and $y \preceq v \preceq z$, where $\theta \in \Theta$.

\section{Main Results}

Our first result is the following coupled coincidence point theorem.

Theorem 2.1. Let $(X, \preceq)$ be a partially ordered set and suppose there is a $G$-metric $G$ on $X$ such that $(X, G)$ is a complete $G$-metric space. Let $F: X \times X \rightarrow X$ and $g: X \rightarrow X$ be mappings such that $F$ has the mixed $g$-monotone property on $X$ and there exist two elements $x_{0}, y_{0} \in X$ with $g\left(x_{0}\right) \preceq F\left(x_{0}, y_{0}\right)$ and $g\left(y_{0}\right) \succeq F\left(y_{0}, x_{0}\right)$. Suppose there exist $\phi \in \Phi$ and $\theta \in \Theta$ such that

$$
\begin{aligned}
& \phi(G(F(x, y), F(u, v), F(w, z)) \\
\leq & \frac{1}{2} \phi(G(g(x), g(u), g(w))+G(g(y), g(v), g(z))) \\
& \quad-\theta(G(g(x), g(u), g(w)), G(g(y), g(v), g(z)))
\end{aligned}
$$

for all $(x, y),(u, v),(w, z) \in X \times X$ with $g(w) \preceq g(u) \preceq g(x)$ and $g(y) \preceq$ $g(v) \preceq g(z)$. Further suppose that $F$ is continuous, $F(X \times X) \subseteq g(X)$, $g$ is continuous and commutes with $F$. Then, there exist $x, y \in X$ such that $F(x, y)=g(x)$ and $F(y, x)=g(y)$, that is, $F$ and $g$ have a coupled coincidence point $(x, y) \in X \times X$. 
Proof. Let $x_{0}, y_{0} \in X$ be such that $g\left(x_{0}\right) \preceq F\left(x_{0}, y_{0}\right)$ and $g\left(y_{0}\right) \succeq F\left(y_{0}, x_{0}\right)$. Since $F(X \times X) \subseteq g(X)$, we can choose $x_{1}, y_{1} \in X$ such that $g\left(x_{1}\right)=$ $F\left(x_{0}, y_{0}\right)$ and $g\left(y_{1}\right)=F\left(y_{0}, x_{0}\right)$.

Analogously, there exist $x_{2}, y_{2} \in X$ such that $g\left(x_{2}\right)=F\left(x_{1}, y_{1}\right)$ and $g\left(y_{2}\right)=F\left(y_{1}, x_{1}\right)$.

Continuing this process, we can construct two sequences $\left\{x_{n}\right\}$ and $\left\{y_{n}\right\}$ in $X$ such that

$$
g\left(x_{n+1}\right)=F\left(x_{n}, y_{n}\right) \text { and } g\left(y_{n+1}\right)=F\left(y_{n}, x_{n}\right) \forall n \geq 0 .
$$

Now we prove that for all $n \geq 0$,

$$
g\left(x_{n}\right) \preceq g\left(x_{n+1}\right) \text { and } g\left(y_{n}\right) \succeq g\left(y_{n+1}\right) .
$$

We shall use the mathematical induction. Let $n=0$, since $g\left(x_{0}\right) \preceq F\left(x_{0}, y_{0}\right)$ and $g\left(y_{0}\right) \succeq F\left(y_{0}, x_{0}\right)$, in view of $g\left(x_{1}\right)=F\left(x_{0}, y_{0}\right)$ and $g\left(y_{1}\right)=F\left(y_{0}, x_{0}\right)$, we have $g\left(x_{0}\right) \preceq g\left(x_{1}\right)$ and $g\left(y_{0}\right) \succeq g\left(y_{1}\right)$, that is, (3) holds for $n=0$. We assume that (3) holds for some $n>0$. As $F$ has the mixed $g$-monotone property and $g\left(x_{n}\right) \preceq g\left(x_{n+1}\right), g\left(y_{n}\right) \succeq g\left(y_{n+1}\right)$, from (2), we get

$$
g\left(x_{n+1}\right)=F\left(x_{n}, y_{n}\right) \preceq F\left(x_{n+1}, y_{n}\right), \quad F\left(y_{n+1}, x_{n}\right) \preceq F\left(y_{n}, x_{n}\right)=g\left(y_{n+1}\right) .
$$

Also for the same reason we have

$g\left(x_{n+2}\right)=F\left(x_{n+1}, y_{n+1}\right) \succeq F\left(x_{n+1}, y_{n}\right), F\left(y_{n+1}, x_{n}\right) \succeq F\left(y_{n+1}, x_{n+1}\right)=g\left(y_{n+2}\right)$.

Merging the above results, we obtain $g\left(x_{n+1}\right) \preceq g\left(x_{n+2}\right)$ and $g\left(y_{n+1}\right) \succeq$ $g\left(y_{n+2}\right)$.

Thus by the mathematical induction, we conclude that (3) holds for all $n \geq 0$.

Since $g\left(x_{n}\right) \succeq g\left(x_{n-1}\right)$ and $g\left(y_{n}\right) \preceq g\left(y_{n-1}\right)$, from (1) and (2), we have

$$
\begin{aligned}
& \phi\left(G\left(g\left(x_{n+1}\right), g\left(x_{n+1}\right), g\left(x_{n}\right)\right)\right) \\
= & \phi\left(G\left(F\left(x_{n}, y_{n}\right), F\left(x_{n}, y_{n}\right), F\left(x_{n-1}, y_{n-1}\right)\right)\right. \\
\leq & \frac{1}{2} \phi\left(G\left(g\left(x_{n}\right), g\left(x_{n}\right), g\left(x_{n-1}\right)\right)+G\left(g\left(y_{n}\right), g\left(y_{n}\right), g\left(y_{n-1}\right)\right)\right) \\
& \quad-\theta\left(G\left(g\left(x_{n}\right), g\left(x_{n}\right), g\left(x_{n-1}\right)\right), G\left(g\left(y_{n}\right), g\left(y_{n}\right), g\left(y_{n-1}\right)\right)\right) .
\end{aligned}
$$

As $\theta\left(t_{1}, t_{2}\right) \geq 0$ for all $t_{1}, t_{2} \in[0,+\infty)$, we have

$$
\begin{aligned}
& \phi\left(G\left(g\left(x_{n+1}\right), g\left(x_{n+1}\right), g\left(x_{n}\right)\right)\right) \\
\leq & \frac{1}{2} \phi\left(G\left(g\left(x_{n}\right), g\left(x_{n}\right), g\left(x_{n-1}\right)\right)+G\left(g\left(y_{n}\right), g\left(y_{n}\right), g\left(y_{n-1}\right)\right)\right) .
\end{aligned}
$$

Similarly, since $g\left(y_{n-1}\right) \succeq g\left(y_{n}\right)$ and $g\left(x_{n-1}\right) \preceq g\left(x_{n}\right)$, from (1) and (2), we have

$$
\begin{aligned}
& \phi\left(G\left(g\left(y_{n+1}\right), g\left(y_{n+1}\right), g\left(y_{n}\right)\right)\right) \\
\leq & \frac{1}{2} \phi\left(G\left(g\left(y_{n}\right), g\left(y_{n}\right), g\left(y_{n-1}\right)\right)+G\left(g\left(x_{n}\right), g\left(x_{n}\right), g\left(x_{n-1}\right)\right)\right) .
\end{aligned}
$$


From (4), (5) and a property of $\phi$, we have

$$
\begin{aligned}
& \phi\left(G\left(g\left(x_{n+1}\right), g\left(x_{n+1}\right), g\left(x_{n}\right)\right)+G\left(g\left(y_{n+1}\right), g\left(y_{n+1}\right), g\left(y_{n}\right)\right)\right) \\
\leq & \frac{1}{2} \phi\left(G\left(g\left(x_{n}\right), g\left(x_{n}\right), g\left(x_{n-1}\right)\right)+G\left(g\left(y_{n}\right), g\left(y_{n}\right), g\left(y_{n-1}\right)\right)\right) \\
& \quad+\frac{1}{2} \phi\left(G\left(g\left(y_{n}\right), g\left(y_{n}\right), g\left(y_{n-1}\right)\right)+G\left(g\left(x_{n}\right), g\left(x_{n}\right), g\left(x_{n-1}\right)\right)\right) \\
= & \phi\left(G\left(g\left(x_{n}\right), g\left(x_{n}\right), g\left(x_{n-1}\right)\right)+G\left(g\left(y_{n}\right), g\left(y_{n}\right), g\left(y_{n-1}\right)\right)\right) .
\end{aligned}
$$

Set $\rho_{n}=G\left(g\left(x_{n+1}\right), g\left(x_{n+1}\right), g\left(x_{n}\right)\right)+G\left(g\left(y_{n+1}\right), g\left(y_{n+1}\right), g\left(y_{n}\right)\right)$, then using the monotonicity of $\phi$ the sequence $\left\{\rho_{n}\right\}$ is non-increasing and so there exists $\rho \geq 0$ such that

$$
\begin{aligned}
\lim _{n \rightarrow+\infty} \rho_{n}= & \lim _{n \rightarrow+\infty}\left[G\left(g\left(x_{n+1}\right), g\left(x_{n+1}\right), g\left(x_{n}\right)\right)\right. \\
& \left.+G\left(g\left(y_{n+1}\right), g\left(y_{n+1}\right), g\left(y_{n}\right)\right)\right] \\
= & \rho .
\end{aligned}
$$

We shall show that $\rho=0$. Suppose, on the contrary, that $\rho>0$. By (6), the sequences $\left\{G\left(g\left(x_{n+1}\right), g\left(x_{n+1}\right), g\left(x_{n}\right)\right)\right\}$ and $\left\{G\left(g\left(y_{n+1}\right), g\left(y_{n+1}\right), g\left(y_{n}\right)\right)\right\}$ have convergent subsequences that are still denoted by $\left\{G\left(g\left(x_{n+1}\right), g\left(x_{n+1}\right), g\left(x_{n}\right)\right)\right\}$ and $\left\{G\left(g\left(y_{n+1}\right), g\left(y_{n+1}\right), g\left(y_{n}\right)\right)\right\}$ respectively. Suppose that

$$
\begin{aligned}
& \lim _{n \rightarrow+\infty} G\left(g\left(x_{n+1}\right), g\left(x_{n+1}\right), g\left(x_{n}\right)\right)=\rho_{1} \text { and } \\
& \lim _{n \rightarrow+\infty} G\left(g\left(y_{n+1}\right), g\left(y_{n+1}\right), g\left(y_{n}\right)\right)=\rho_{2} .
\end{aligned}
$$

Then $\rho_{1}+\rho_{2}=\rho>0$. Reasoning as above and using a property of $\phi$, we have

$$
\begin{aligned}
& \phi\left(G\left(g\left(x_{n+1}\right), g\left(x_{n+1}\right), g\left(x_{n}\right)\right)+G\left(g\left(y_{n+1}\right), g\left(y_{n+1}\right), g\left(y_{n}\right)\right)\right) \\
& \leq \phi\left(G\left(g\left(x_{n+1}\right), g\left(x_{n+1}\right), g\left(x_{n}\right)\right)\right)+\phi\left(G\left(g\left(y_{n+1}\right), g\left(y_{n+1}\right), g\left(y_{n}\right)\right)\right) \\
& \leq \phi\left(G\left(g\left(x_{n}\right), g\left(x_{n}\right), g\left(x_{n-1}\right)\right)+G\left(g\left(y_{n}\right), g\left(y_{n}\right), g\left(y_{n-1}\right)\right)\right) \\
& \quad-\theta\left(G\left(g\left(x_{n}\right), g\left(x_{n}\right), g\left(x_{n-1}\right)\right), G\left(g\left(y_{n}\right), g\left(y_{n}\right), g\left(y_{n-1}\right)\right)\right) \\
& \quad-\theta\left(G\left(g\left(y_{n}\right), g\left(y_{n}\right), g\left(y_{n-1}\right)\right), G\left(g\left(x_{n}\right), g\left(x_{n}\right), g\left(x_{n-1}\right)\right)\right) .
\end{aligned}
$$

Taking the limit as $n \rightarrow+\infty$ in the last inequality, using (6), the continuity of $\phi$ and the property of $\theta$, we have

$$
\begin{aligned}
\phi(\rho) \leq & \phi(\rho)-\lim _{n \rightarrow+\infty} \theta\left(G\left(g\left(x_{n}\right), g\left(x_{n}\right), g\left(x_{n-1}\right)\right), G\left(g\left(y_{n}\right), g\left(y_{n}\right), g\left(y_{n-1}\right)\right)\right) \\
& \quad-\lim _{n \rightarrow+\infty} \theta\left(G\left(g\left(y_{n}\right), g\left(y_{n}\right), g\left(y_{n-1}\right)\right), G\left(g\left(x_{n}\right), g\left(x_{n}\right), g\left(x_{n-1}\right)\right)\right) \\
<\phi(\rho) &
\end{aligned}
$$


which is a contradiction. Thus $\rho=0$, that is,

$$
\begin{aligned}
& \lim _{n \rightarrow+\infty} G\left(g\left(x_{n+1}\right), g\left(x_{n+1}\right), g\left(x_{n}\right)\right)=0, \\
& \lim _{n \rightarrow+\infty} G\left(g\left(y_{n+1}\right), g\left(y_{n+1}\right), g\left(y_{n}\right)\right)=0 .
\end{aligned}
$$

In what follows, we shall prove that $\left\{g\left(x_{n}\right)\right\}$ and $\left\{g\left(y_{n}\right)\right\}$ are Cauchy sequences. Suppose, to the contrary, that at least one of $\left\{g\left(x_{n}\right)\right\}$ and $\left\{g\left(y_{n}\right)\right\}$ is not a $G$-Cauchy sequence, that is,

$$
\lim _{m, n \rightarrow+\infty} G\left(g\left(x_{m}\right), g\left(x_{n}\right), g\left(x_{n}\right)\right) \neq 0 .
$$

Then, there exists $\varepsilon>0$ for which we can find two subsequences $\left\{g\left(x_{m(i)}\right)\right\}$ and $\left\{g\left(x_{n(i)}\right)\right\}$ of $\left\{x_{n}\right\}$ such that $n(i)$ is the smallest index for which $n(i)>$ $m(i)>i$,

$$
r_{i}=G\left(g\left(x_{n(i)}\right), g\left(x_{n(i)}\right), g\left(x_{m(i)}\right)\right)+G\left(g\left(y_{n(i)}\right), g\left(y_{n(i)}\right), g\left(y_{m(i)}\right)\right) \geq \varepsilon .
$$

This means that

$$
G\left(g\left(x_{n(i)-1}\right), g\left(x_{n(i)-1}\right), g\left(x_{m(i)}\right)\right)+G\left(g\left(y_{n(i)-1}\right), g\left(y_{n(i)-1}\right), g\left(y_{m(i)}\right)\right)<\varepsilon .
$$

By rectangle inequality, we get

$$
\begin{aligned}
& G\left(g\left(x_{n(i)}\right), g\left(x_{n(i)}\right), g\left(x_{m(i)}\right)\right) \\
\leq & G\left(g\left(x_{n(i)}\right), g\left(x_{n(i)}\right), g\left(x_{n(i)-1}\right)\right)+G\left(g\left(x_{n(i)-1}\right), g\left(x_{n(i)-1}\right), g\left(x_{m(i)}\right)\right)
\end{aligned}
$$

and

$$
\begin{aligned}
& G\left(g\left(y_{n(i)}\right), g\left(y_{n(i)}\right), g\left(y_{m(i)}\right)\right) \\
\leq & G\left(g\left(y_{n(i)}\right), g\left(y_{n(i)}\right), g\left(y_{n(i)-1}\right)\right)+G\left(g\left(y_{n(i)-1}\right), g\left(y_{n(i)-1}\right), g\left(y_{m(i)}\right)\right) .
\end{aligned}
$$

Using the above inequalities, we get

$$
\begin{aligned}
\varepsilon & \leq r_{i}=G\left(g\left(x_{n(i)}\right), g\left(x_{n(i)}\right), g\left(x_{m(i)}\right)\right)+G\left(g\left(y_{n(i)}\right), g\left(y_{n(i)}\right), g\left(y_{m(i)}\right)\right) \\
& \leq G\left(g\left(x_{n(i)}\right), g\left(x_{n(i)}\right), g\left(x_{n(i)-1}\right)\right)+G\left(g\left(y_{n(i)}\right), g\left(y_{n(i)}\right), g\left(y_{n(i)-1}\right)\right)+\varepsilon .
\end{aligned}
$$

Letting $i \rightarrow+\infty$ and using (7) we have

(8)

$$
\lim _{i \rightarrow+\infty} r_{i}=\lim _{i \rightarrow+\infty}\left[G\left(g\left(x_{n(i)}\right), g\left(x_{n(i)}\right), g\left(x_{m(i)}\right)\right)\right.
$$$$
\left.+G\left(g\left(y_{n(i)}\right), g\left(y_{n(i)}\right), g\left(y_{m(i)}\right)\right)\right]
$$ 
Using the fact that $G(x, x, y) \leq 2 G(x, y, y)$ for any $x, y \in X$, we obtain from (G5) that

$$
\begin{aligned}
& \quad G\left(g\left(x_{n(i)}\right), g\left(x_{n(i)}\right), g\left(x_{m(i)}\right)\right) \\
& \leq G\left(g\left(x_{n(i)}\right), g\left(x_{n(i)}\right), g\left(x_{n(i)+1}\right)\right) \\
& \quad+G\left(g\left(x_{n(i)+1}\right), g\left(x_{n(i)+1}\right), g\left(x_{m(i)}\right)\right) \\
& \leq 2 G\left(g\left(x_{n(i)+1}\right), g\left(x_{n(i)+1}\right), g\left(x_{n(i)}\right)\right) \\
& \quad+G\left(g\left(x_{n(i)+1}\right), g\left(x_{n(i)+1}\right), g\left(x_{m(i)+1}\right)\right) \\
& \quad+G\left(g\left(x_{m(i)+1}\right), g\left(x_{m(i)+1}\right), g\left(x_{m(i)}\right)\right)
\end{aligned}
$$

and

$$
\begin{aligned}
& \quad G\left(g\left(y_{n(i)}\right), g\left(y_{n(i)}\right), g\left(y_{m(i)}\right)\right) \\
& \leq 2 G\left(g\left(y_{n(i)+1}\right), g\left(y_{n(i)+1}\right), g\left(y_{n(i)}\right)\right) \\
& \quad+G\left(g\left(y_{n(i)+1}\right), g\left(y_{n(i)+1}\right), g\left(y_{m(i)+1}\right)\right) \\
& \quad+G\left(g\left(y_{m(i)+1}\right), g\left(y_{m(i)+1}\right), g\left(y_{m(i)}\right)\right) .
\end{aligned}
$$

By (9) and (10), we have

$$
\begin{gathered}
r_{i}=G\left(g\left(x_{n(i)}\right), g\left(x_{n(i)}\right), g\left(x_{m(i)}\right)\right)+G\left(g\left(y_{n(i)}\right), g\left(y_{n(i)}\right), g\left(y_{m(i)}\right)\right) \\
\leq 2 \rho_{n(i)}+\rho_{m(i)}+G\left(g\left(x_{n(i)+1}\right), g\left(x_{n(i)+1}\right), g\left(x_{m(i)+1}\right)\right) \\
+G\left(g\left(y_{n(i)+1}\right), g\left(y_{n(i)+1}\right), g\left(y_{m(i)+1}\right)\right) .
\end{gathered}
$$

By (11), using the properties of $\phi$, we get

$$
\begin{gathered}
\phi\left(r_{i}\right) \leq \phi\left(2 \rho_{n(i)}+\rho_{m(i)}+G\left(g\left(x_{n(i)+1}\right), g\left(x_{n(i)+1}\right), g\left(x_{m(i)+1}\right)\right)\right. \\
\left.+G\left(g\left(y_{n(i)+1}\right), g\left(y_{n(i)+1}\right), g\left(y_{m(i)+1}\right)\right)\right) \\
\leq 2 \phi\left(\rho_{n(i)}\right)+\phi\left(\rho_{m(i)}\right)+\phi\left(G\left(g\left(x_{n(i)+1}\right), g\left(x_{n(i)+1}\right), g\left(x_{m(i)+1}\right)\right)\right) \\
+\phi\left(G\left(g\left(y_{n(i)+1}\right), g\left(y_{n(i)+1}\right), g\left(y_{m(i)+1}\right)\right)\right) .
\end{gathered}
$$

Since $n(i)>m(i), g\left(x_{n(i)}\right) \succeq g\left(x_{m(i)}\right)$ and $g\left(y_{m(i)}\right) \succeq g\left(y_{n(i)}\right)$, by (1) we deduce

$$
\begin{aligned}
& \phi\left(G\left(g\left(x_{n(i)+1}\right), g\left(x_{n(i)+1}\right), g\left(x_{m(i)+1}\right)\right)\right) \\
= & \phi\left(G\left(F\left(x_{n(i)}, y_{n(i)}\right), F\left(x_{n(i)}, y_{n(i)}\right), F\left(x_{m(i)}, y_{m(i)}\right)\right)\right. \\
\leq & \frac{1}{2} \phi\left(G\left(g\left(x_{n(i)}\right), g\left(x_{n(i)}\right), g\left(x_{m(i)}\right)\right)+G\left(g\left(y_{n(i)}\right), g\left(y_{n(i)}\right), g\left(y_{m(i)}\right)\right)\right) \\
\quad & \quad-\theta\left(G\left(g\left(x_{n(i)}\right), g\left(x_{n(i)}\right), g\left(x_{m(i)}\right)\right), G\left(g\left(y_{n(i)}\right), g\left(y_{n(i)}\right), g\left(y_{m(i)}\right)\right)\right) \\
\leq & \frac{1}{2} \phi\left(r_{i}\right)-\theta\left(G\left(g\left(x_{n(i)}\right), g\left(x_{n(i)}\right), g\left(x_{m(i)}\right)\right), G\left(g\left(y_{n(i)}\right), g\left(y_{n(i)}\right), g\left(y_{m(i)}\right)\right)\right)
\end{aligned}
$$


Similarly, we have

$$
\begin{aligned}
& \phi\left(G\left(g\left(y_{n(i)+1}\right), g\left(y_{n(i)+1}\right), g\left(y_{m(i)+1}\right)\right)\right) \leq \frac{1}{2} \phi\left(r_{i}\right) \\
& \quad-\theta\left(G\left(g\left(y_{n(i)}\right), g\left(y_{n(i)}\right), g\left(y_{m(i)}\right)\right), G\left(g\left(x_{n(i)}\right), g\left(x_{n(i)}\right), g\left(x_{m(i)}\right)\right)\right) .
\end{aligned}
$$

Inserting (13) and (14) in (12), we get

$$
\begin{aligned}
& \phi\left(r_{i}\right) \leq 2 \phi\left(\rho_{n(i)}\right)+\phi\left(\rho_{m(i)}\right)+\phi\left(r_{i}\right) \\
& \quad-\theta\left(G\left(g\left(x_{n(i)}\right), g\left(x_{n(i)}\right), g\left(x_{m(i)}\right)\right), G\left(g\left(y_{n(i)}\right), g\left(y_{n(i)}\right), g\left(y_{m(i)}\right)\right)\right) \\
& \quad-\theta\left(G\left(g\left(y_{n(i)}\right), g\left(y_{n(i)}\right), g\left(y_{m(i)}\right)\right), G\left(g\left(x_{n(i)}\right), g\left(x_{n(i)}\right), g\left(x_{m(i)}\right)\right)\right) .
\end{aligned}
$$

By (8), the sequences

$$
\left\{G\left(g\left(x_{n(i)}\right), g\left(x_{n(i)}\right), g\left(x_{m(i)}\right)\right)\right\} \quad \text { and } \quad\left\{G\left(g\left(y_{n(i)}\right), g\left(y_{n(i)}\right), g\left(y_{m(i)}\right)\right)\right\}
$$

have subsequences converging to $\varepsilon_{1}$ and $\varepsilon_{2}$ (say) respectively and $\varepsilon_{1}+\varepsilon_{2}=$ $\varepsilon>0$. By passing to subsequences, we may assume that

$$
\lim _{i \rightarrow+\infty} G\left(g\left(x_{n(i)}\right), g\left(x_{n(i)}\right), g\left(x_{m(i)}\right)\right)=\varepsilon_{1}
$$

and

$$
\lim _{i \rightarrow+\infty} G\left(g\left(y_{n(i)}\right), g\left(y_{n(i)}\right), g\left(y_{m(i)}\right)\right)=\varepsilon_{2} .
$$

Taking $i \rightarrow+\infty$ in (15) and using (7), (8), the properties of $\phi$ and $\theta$, we have

$$
\begin{aligned}
& \phi(\varepsilon) \leq 2 \phi(0)+\phi(0)+\phi(\varepsilon)-\lim _{i \rightarrow+\infty} \theta\left(G\left(g\left(x_{n(i)}\right), g\left(x_{n(i)}\right), g\left(x_{m(i)}\right)\right),\right. \\
&\left.G\left(g\left(y_{n(i)}\right), g\left(y_{n(i)}\right), g\left(y_{m(i)}\right)\right)\right) \\
&-\lim _{i \rightarrow+\infty} \theta\left(G\left(g\left(y_{n(i)}\right), g\left(y_{n(i)}\right), g\left(y_{m(i)}\right)\right), G\left(g\left(x_{n(i)}\right), g\left(x_{n(i)}\right), g\left(x_{m(i)}\right)\right)\right) \\
&=\phi(\varepsilon)-\lim _{i \rightarrow+\infty} \theta\left(G\left(g\left(x_{n(i)}\right), g\left(x_{n(i)}\right), g\left(x_{m(i)}\right)\right),\right. \\
&\left.G\left(g\left(y_{n(i)}\right), g\left(y_{n(i)}\right), g\left(y_{m(i)}\right)\right)\right) \\
&-\lim _{i \rightarrow+\infty} \theta\left(G\left(g\left(y_{n(i)}\right), g\left(y_{n(i)}\right), g\left(y_{m(i)}\right)\right), G\left(g\left(x_{n(i)}\right), g\left(x_{n(i)}\right), g\left(x_{m(i)}\right)\right)\right) \\
&<\phi(\varepsilon),
\end{aligned}
$$

which is a contradiction. Thus, $\left\{g\left(x_{n}\right)\right\}$ and $\left\{g\left(y_{n}\right)\right\}$ are Cauchy sequences. Since the $G$-metric space $(X, G)$ is complete, there exist $x, y \in X$ such that $\left\{g\left(x_{n}\right)\right\}$ and $\left\{g\left(y_{n}\right)\right\}$ are convergent to $x$ and $y$ respectively, that is from Proposition 1.1, we have

$$
\lim _{i \rightarrow+\infty} G\left(g\left(x_{n}\right), g\left(x_{n}\right), x\right)=\lim _{i \rightarrow+\infty} G\left(g\left(x_{n}\right), x, x\right)=0
$$

and

$$
\lim _{i \rightarrow+\infty} G\left(g\left(y_{n}\right), g\left(y_{n}\right), y\right)=\lim _{i \rightarrow+\infty} G\left(g\left(y_{n}\right), y, y\right)=0 .
$$


Using the continuity of $g$ and Proposition 1.3, we get

$$
\lim _{i \rightarrow+\infty} G\left(g\left(g\left(x_{n}\right)\right), g\left(g\left(x_{n}\right)\right), g(x)\right)=\lim _{i \rightarrow+\infty} G\left(g\left(g\left(x_{n}\right)\right), g(x), g(x)\right)=0
$$

and

$$
\lim _{i \rightarrow+\infty} G\left(g\left(g\left(y_{n}\right)\right), g\left(g\left(y_{n}\right)\right), g(y)\right)=\lim _{i \rightarrow+\infty} G\left(g\left(g\left(y_{n}\right)\right), g(y), g(y)\right)=0 .
$$

Since, $g\left(x_{n+1}\right)=F\left(x_{n}, y_{n}\right)$ and $g\left(y_{n+1}\right)=F\left(y_{n}, x_{n}\right)$, hence the commutativity of $F$ and $g$ yields that

$$
\left\{\begin{array}{l}
g\left(g\left(x_{n+1}\right)\right)=g\left(F\left(x_{n}, y_{n}\right)\right)=F\left(g\left(x_{n}\right), g\left(y_{n}\right)\right), \\
g\left(g\left(y_{n+1}\right)\right)=g\left(F\left(y_{n}, x_{n}\right)\right)=F\left(g\left(y_{n}\right), g\left(x_{n}\right)\right) .
\end{array}\right.
$$

Next we show that $F(x, y)=g(x)$ and $F(y, x)=g(y)$. The mapping $F$ is continuous and since the sequences $\left\{g\left(x_{n}\right)\right\}$ and $\left\{g\left(y_{n}\right)\right\}$ are respectively $G$-convergent to $x$ and $y$, hence using Definition 1.3 the sequence $\left\{F\left(g\left(x_{n}\right), g\left(y_{n}\right)\right)\right\}$ is $G$-convergent to $F(x, y)$. Therefore, by $(17),\left\{g\left(g\left(x_{n+1}\right)\right)\right\}$ is $G$-convergent to $F(x, y)$. By uniqueness of limit and using (16), we have $F(x, y)=g(x)$. Similarly, we can show that $F(y, x)=g(y)$. Hence $(x, y)$ is a coupled coincidence point of $F$ and $g$.

Theorem 2.2. Let $(X, \preceq)$ be a partially ordered set and $G$ be a $G$-metric on $X$. Let $F: X \times X \rightarrow X$ and $g: X \rightarrow X$ be mappings such that $F$ has the mixed $g$-monotone property on $X$ and there exist two elements $x_{0}, y_{0} \in X$ with $g\left(x_{0}\right) \preceq F\left(x_{0}, y_{0}\right)$ and $g\left(y_{0}\right) \succeq F\left(y_{0}, x_{0}\right)$. Suppose there exist $\phi \in \Phi$ and $\theta \in \Theta$ such that

$$
\begin{aligned}
& \phi(G(F(x, y), F(u, v), F(w, z)) \\
\leq & \frac{1}{2} \phi(G(g(x), g(u), g(w))+G(g(y), g(v), g(z))) \\
& \quad-\theta(G(g(x), g(u), g(w)), G(g(y), g(v), g(z)))
\end{aligned}
$$

for all $(x, y),(u, v),(w, z) \in X \times X$ with $g(w) \preceq g(u) \preceq g(x)$ and $g(y) \preceq$ $g(v) \preceq g(z)$. Further suppose that $(g(X), G)$ or $(F(X \times X), G)$ is complete, $F(X \times X) \subseteq g(X)$ and the following conditions hold:

(i) if a non-decreasing sequence $\left\{x_{n}\right\}$ in $X$ converges to $x \in X$, then $x_{n} \preceq x$ for all $n$,

(ii) if a non-increasing sequence $\left\{y_{n}\right\}$ in $X$ converges to $y \in X$, then $y_{n} \succeq y$ for all $n$.

Then, there exist $x, y \in X$ such that $F(x, y)=g(x)$ and $F(y, x)=g(y)$, that is, $F$ and $g$ have a coupled coincidence point $(x, y) \in X \times X$.

Proof. Following the proof of Theorem 2.1, it follows that $\left\{g\left(x_{n}\right)\right\}$ and $\left\{g\left(y_{n}\right)\right\}$ are Cauchy sequences. Now, we distinguish the following two cases. Case 1. If $(g(X), G)$ is complete, then there exist $x, y \in X$ such that $g\left(x_{n}\right) \rightarrow g(x)$ and $g\left(y_{n}\right) \rightarrow g(y)$ as $n \rightarrow+\infty$. Since $\left\{g\left(x_{n}\right)\right\}$ is nondecreasing and $\left\{g\left(y_{n}\right)\right\}$ is non-increasing, by given hypotheses, we have 
$g\left(x_{n}\right) \preceq g(x)$ and $g(y) \preceq g\left(y_{n}\right)$ for all $n \geq 0$. Then using (18) and the properties of $\phi$ and $\theta$, we have

$$
\begin{aligned}
& \phi\left(G\left(F(x, y), g\left(x_{n+1}\right), g\left(x_{n+1}\right)\right)\right) \\
= & \phi\left(G\left(F(x, y), F\left(x_{n}, y_{n}\right), F\left(x_{n}, y_{n}\right)\right)\right) \\
\leq & \frac{1}{2} \phi\left(G\left(g(x), g\left(x_{n}\right), g\left(x_{n}\right)\right)+G\left(g(y), g\left(y_{n}\right), g\left(y_{n}\right)\right)\right) \\
& \quad-\theta\left(G\left(g(x), g\left(x_{n}\right), g\left(x_{n}\right)\right), G\left(g(y), g\left(y_{n}\right), g\left(y_{n}\right)\right)\right) \\
\leq & \frac{1}{2} \phi\left(G\left(g(x), g\left(x_{n}\right), g\left(x_{n}\right)\right)+G\left(g(y), g\left(y_{n}\right), g\left(y_{n}\right)\right)\right) .
\end{aligned}
$$

Letting $n \rightarrow+\infty$ in the last inequality and using the properties of $\phi$, we obtain

$$
\phi(G(F(x, y), g(x), g(x))) \leq 0,
$$

which implies that $G(F(x, y), g(x), g(x))=0$, that is, $F(x, y)=g(x)$. Similarly, it can be shown that $F(y, x)=g(y)$.

Case 2. If $(F(X \times X), G)$ is complete, then there exist $p, q \in F(X \times X)$ such that $F\left(x_{n}, y_{n}\right) \rightarrow p$ and $F\left(y_{n}, x_{n}\right) \rightarrow q$ as $n \rightarrow+\infty$. Since $F(X \times X) \subseteq$ $g(X)$, so there exist $x, y \in X$ such that $p=g(x)$ and $q=g(y)$ and from here onwards the proof follows as in Case 1.

If $g=I$, the identity mapping in Theorem 2.1, then we deduce the following result of coupled fixed point.

Corollary 2.1. Let $(X, \preceq)$ be a partially ordered set and suppose there is a $G$-metric $G$ on $X$ such that $(X, G)$ is a complete $G$-metric space. Let $F$ : $X \times X \rightarrow X$ be a continuous mapping such that $F$ has the mixed monotone property on $X$ and there exist two elements $x_{0}, y_{0} \in X$ with $x_{0} \preceq F\left(x_{0}, y_{0}\right)$ and $y_{0} \succeq F\left(y_{0}, x_{0}\right)$. Also suppose there exist $\phi \in \Phi$ and $\theta \in \Theta$ such that

$$
\begin{aligned}
& \phi(G(F(x, y), F(u, v), F(w, z)) \\
\leq & \frac{1}{2} \phi(G(x, u, w)+G(y, v, z))-\theta(G(x, u, w), G(y, v, z))
\end{aligned}
$$

for all $(x, y),(u, v),(w, z) \in X \times X$ with $w \preceq u \preceq x$ and $y \preceq v \preceq z$ Then, there exist $x, y \in X$ such that $F(x, y)=x$ and $F(y, x)=y$, that is, $F$ has a coupled fixed point $(x, y) \in X \times X$.

If $\phi=g=I$, the identity mapping in Theorem 2.1, then we obtain the result of Luong and Thuan [9] in the form of following corollary.

Corollary $2.2([9])$. Let $(X, \preceq)$ be a partially ordered set and suppose there is a $G$-metric $G$ on $X$ such that $(X, G)$ is a complete $G$-metric space. Let $F: X \times X \rightarrow X$ be a mapping such that $F$ is continuous and has the mixed monotone property on $X$ and there exist two elements $x_{0}, y_{0} \in X$ with 
$x_{0} \preceq F\left(x_{0}, y_{0}\right)$ and $y_{0} \succeq F\left(y_{0}, x_{0}\right)$. Also suppose there exists $\theta \in \Theta$ such that

$$
\begin{aligned}
& G(F(x, y), F(u, v), F(w, z) \\
\leq & \frac{1}{2}(G(x, u, w)+G(y, v, z))-\theta(G(x, u, w), G(y, v, z))
\end{aligned}
$$

for all $(x, y),(u, v),(w, z) \in X \times X$ with $w \preceq u \preceq x$ and $y \preceq v \preceq z$. Then, there exist $x, y \in X$ such that $F(x, y)=x$ and $F(y, x)=y$, that is, $F$ has a coupled fixed point $(x, y) \in X \times X$.

Let $\Psi$ denote the class of functions $\psi:[0,+\infty) \rightarrow[0,+\infty)$ satisfying $\lim _{t \rightarrow r} \psi(t)>0$ for each $r>0$. Now, considering $\theta\left(t_{1}, t_{2}\right)=\psi\left(\max \left\{t_{1}, t_{2}\right\}\right)$ for all $t_{1}, t_{2} \in[0,+\infty)$ with $\psi \in \Psi$ in Theorem 2.1, we have the following corollary.

Corollary 2.3. Let $(X, \preceq)$ be a partially ordered set and suppose there is a $G$-metric $G$ on $X$ such that $(X, G)$ is a complete $G$-metric space. Let $F: X \times X \rightarrow X$ and $g: X \times X$ be mappings such that $F$ is continuous, $F$ has the g-mixed monotone property on $X$ and there exist two elements $x_{0}, y_{0} \in X$ with $g\left(x_{0}\right) \preceq F\left(x_{0}, y_{0}\right)$ and $g\left(y_{0}\right) \succeq F\left(y_{0}, x_{0}\right)$. Also suppose there exist $\phi \in \Phi$ and $\psi \in \Psi$ such that

$$
\begin{aligned}
& \phi(G(F(x, y), F(u, v), F(w, z)) \\
\leq & \frac{1}{2} \phi(G(g(x), g(u), g(w))+G(g(y), g(v), g(z))) \\
- & \psi(\max \{G(g(x), g(u), g(w)), G(g(y), g(v), g(z))\})
\end{aligned}
$$

for all $(x, y),(u, v),(w, z) \in X \times X$ with $g(w) \preceq g(u) \preceq g(x)$ and $g(y) \preceq$ $g(v) \preceq g(z)$. Then, there exist $x, y \in X$ such that $F(x, y)=g(x)$ and $F(y, x)=g(y)$, that is, $F$ and $g$ have a coupled coincidence point $(x, y) \in$ $X \times X$.

Moreover, if $\phi=g=I$, the identity mapping in Theorem 2.1, $\theta\left(t_{1}, t_{2}\right)=$ $\frac{1-k}{2}\left(t_{1}+t_{2}\right)$ for all $t_{1}, t_{2} \in[0,+\infty)$, where $\theta \in \Theta$ with $k \in[0,1)$ then we obtain the main result of Choudhury and Maity [5] in the form of the following corollary.

Corollary 2.4. Let $(X, \preceq)$ be a partially ordered set and suppose there is a $G$-metric $G$ on $X$ such that $(X, G)$ is a complete $G$-metric space. Let $F: X \times X \rightarrow X$ be a mapping such that $F$ is continuous and has the mixed monotone property on $X$ and there exist two elements $x_{0}, y_{0} \in X$ with $x_{0} \preceq F\left(x_{0}, y_{0}\right)$ and $y_{0} \succeq F\left(y_{0}, x_{0}\right)$. Also suppose there exists $k \in[0,1)$ such that

$$
G\left(F(x, y), F(u, v), F(w, z) \leq \frac{k}{2}(G(x, u, w)+G(y, v, z))\right.
$$

for all $(x, y),(u, v),(w, z) \in X \times X$ with $w \preceq u \preceq x$ and $y \preceq v \preceq z$. Then, there exist $x, y \in X$ such that $F(x, y)=x$ and $F(y, x)=y$, that is, $F$ has a coupled fixed point $(x, y) \in X \times X$. 
Now, we give sufficient conditions for uniqueness of the coupled fixed point. If $(X, \preceq)$ is a partially ordered set, then we endow the product space $X \times X$ with the following partial order:

$$
\text { for }(x, y),(u, v) \in X \times X, \quad(u, v) \preceq(x, y) \Leftrightarrow x \succeq u, y \preceq v .
$$

Theorem 2.3. In addition to the hypotheses of Theorem 2.1, suppose that for every $(x, y),\left(x^{*}, y^{*}\right) \in X \times X$ there exists $(u, v) \in X \times X$ such that $(F(u, v), F(v, u))$ is comparable to $(F(x, y), F(y, x))$ and $\left(F\left(x^{*}, y^{*}\right), F\left(y^{*}, x^{*}\right)\right)$. Then $F$ and $g$ have a unique coupled common fixed point, that is, there exists a unique $(x, y) \in X \times X$ such that $x=g(x)=F(x, y)$ and $y=g(y)=$ $F(y, x)$.

Proof. From Theorem 2.1, the set of coupled coincidence points of $F$ and $g$ is non-empty. Suppose that $(x, y)$ and $\left(x^{*}, y^{*}\right)$ are coupled coincidence points of $F$ and $g$, that is, $g(x)=F(x, y), g(y)=F(y, x), g\left(x^{*}\right)=F\left(x^{*}, y^{*}\right)$ and $g\left(y^{*}\right)=F\left(y^{*}, x^{*}\right)$, then we show that

$$
g(x)=g\left(x^{*}\right) \text { and } g(y)=g\left(y^{*}\right) .
$$

By assumption, there exists $(u, v) \in X \times X$ such that $(F(u, v), F(v, u))$ is comparable to $(F(x, y), F(y, x))$ and $\left(F\left(x^{*}, y^{*}\right), F\left(y^{*}, x^{*}\right)\right)$. Put $u_{0}=u$, $v_{0}=v$, and choose $u_{1}, v_{1} \in X$ so that $g\left(u_{1}\right)=F\left(u_{0}, v_{0}\right)$ and $g\left(v_{1}\right)=$ $F\left(v_{0}, u_{0}\right)$. Then, proceeding as in the proof of Theorem 2.1, we can inductively define sequences $\left\{g\left(u_{n}\right)\right\},\left\{g\left(v_{n}\right)\right\}$ such that

$$
g\left(u_{n+1}\right)=F\left(u_{n}, v_{n}\right) \text { and } g\left(v_{n+1}\right)=F\left(v_{n}, u_{n}\right) \forall n \geq 0 .
$$

Further, set $x_{0}=x, y_{0}=y, x_{0}^{*}=x^{*}, y_{0}^{*}=y^{*}$ and, on the same way, define the sequences $\left\{g\left(x_{n}\right)\right\},\left\{g\left(y_{n}\right)\right\},\left\{g\left(x_{n}^{*}\right)\right\}$ and $\left\{g\left(y_{n}^{*}\right)\right\}$. Then it is easy to show that

$$
\begin{array}{ll}
g\left(x_{n}\right) \rightarrow F(x, y), & g\left(y_{n}\right) \rightarrow F(y, x), \\
g\left(x_{n}^{*}\right) \rightarrow F\left(x^{*}, y^{*}\right), & g\left(y_{n}^{*}\right) \rightarrow F\left(y^{*}, x^{*}\right)
\end{array}
$$

as $n \rightarrow+\infty$.

Since $(F(x, y), F(y, x))=\left(g\left(x_{1}\right), g\left(y_{1}\right)\right)=(g(x), g(y))$ and $(F(u, v), F(v, u))=$ $\left(g\left(u_{1}\right), g\left(v_{1}\right)\right)$ are comparable, then $g(x) \preceq g\left(u_{1}\right)$ and $g(y) \succeq g\left(v_{1}\right)$. It is easy to show that $(g(x), g(y))$ and $\left(g\left(u_{n}\right), g\left(v_{n}\right)\right)$ are comparable, that is, $g(x) \preceq g\left(u_{n}\right)$ and $g(y) \succeq g\left(v_{n}\right)$ for all $n \geq 1$. Thus from (1), we have

$$
\begin{aligned}
& \phi\left(G\left(g\left(u_{n+1}\right), g(x), g(x)\right)\right) \\
= & \phi\left(G\left(F\left(u_{n}, v_{n}\right), F(x, y), F(x, y)\right)\right) \\
\leq & \frac{1}{2} \phi\left(G\left(g\left(u_{n}\right), g(x), g(x)\right)+G\left(g v_{n}, g(y), g(y)\right)\right) \\
& \quad-\theta\left(G\left(g\left(u_{n}\right), g(x), g(x)\right), G\left(g\left(v_{n}\right), g(y), g(y)\right)\right) .
\end{aligned}
$$


Similarly,

$$
\begin{aligned}
& \phi\left(G\left(g\left(v_{n+1}\right), g(y), g(y)\right)\right) \\
= & \phi\left(G\left(F\left(v_{n}, u_{n}\right), F(y, x), F(y, x)\right)\right) \\
\leq & \frac{1}{2} \phi\left(G\left(g\left(v_{n}\right), g(y), g(y)\right)+G\left(g\left(u_{n}\right), g(x), g(x)\right)\right) \\
\quad & \quad-\theta\left(G\left(g\left(v_{n}\right), g(y), g(y)\right), G\left(g\left(u_{n}\right), g(x), g(x)\right)\right) .
\end{aligned}
$$

Using the above inequalities and a property of $\phi$, we have

$$
\begin{aligned}
& \phi\left(G\left(g\left(u_{n+1}\right), g(x), g(x)\right)+G\left(g\left(v_{n+1}\right), g(y), g(y)\right)\right) \\
\leq & \phi\left(G\left(g\left(u_{n+1}\right), g(x), g(x)\right)\right)+\phi\left(G\left(g\left(v_{n+1}\right), g(y), g(y)\right)\right) \\
\leq & \phi\left(G\left(g\left(u_{n}\right), g(x), g(x)\right)+G\left(g\left(v_{n}\right), g(y), g(y)\right)\right) \\
& \quad-\theta\left(G\left(g\left(u_{n}\right), g(x), g(x)\right), G\left(g\left(v_{n}\right), g(y), g(y)\right)\right) \\
& \quad-\theta\left(G\left(g\left(v_{n}\right), g(y), g(y)\right), G\left(g\left(u_{n}\right), g(x), g(x)\right)\right) \\
\leq & \phi\left(G\left(g\left(u_{n}\right), g(x), g(x)\right)+G\left(g\left(v_{n}\right), g(y), g(y)\right)\right) .
\end{aligned}
$$

By monotonicity of $\phi$, it follows that

$$
\begin{aligned}
& G\left(g\left(u_{n+1}\right), g(x), g(x)\right)+G\left(g\left(v_{n+1}\right), g(y), g(y)\right) \\
\leq & G\left(g\left(u_{n}\right), g(x), g(x)\right)+G\left(g\left(v_{n}\right), g(y), g(y)\right) .
\end{aligned}
$$

Let $\alpha_{n}=G\left(g\left(u_{n}\right), g(x), g(x)\right)+G\left(g\left(v_{n}\right), g(y), g(y)\right)$, then the sequence $\left\{\alpha_{n}\right\}$ is a non-increasing sequence, so there exists some $\alpha \geq 0$ such that

$$
\lim _{n \rightarrow+\infty} \alpha_{n}=\lim _{n \rightarrow+\infty}\left[G\left(g\left(u_{n}\right), g(x), g(x)\right)+G\left(g\left(v_{n}\right), g(y), g(y)\right)\right]=\alpha .
$$

We shall show that $\alpha=0$. Suppose, to the contrary, that $\alpha>0$. Therefore, $\left\{G\left(g\left(u_{n}\right), g(x), g(x)\right)\right\}$ and $\left\{G\left(g\left(v_{n}\right), g(y), g(y)\right)\right\}$ have subsequences converging to $\alpha_{1}$ and $\alpha_{2}$ (say) respectively. Taking the limit, up to subsequences, as $n \rightarrow+\infty$ in (21) and using the continuity of $\phi$, we have

$$
\begin{aligned}
\phi(\alpha) \leq \phi(\alpha)- & \lim _{n \rightarrow+\infty} \theta\left(G\left(g\left(u_{n}\right), g(x), g(x)\right), G\left(g\left(v_{n}\right), g(y), g(y)\right)\right) \\
& -\lim _{n \rightarrow+\infty} \theta\left(G\left(g\left(v_{n}\right), g(y), g(y)\right), G\left(g\left(u_{n}\right), g(x), g(x)\right)\right) \\
& <\phi(\alpha),
\end{aligned}
$$

a contradiction. Thus, $\alpha=0$, that is

$$
\lim _{n \rightarrow+\infty}\left[G\left(g\left(u_{n}\right), g(x), g(x)\right)+G\left(g\left(v_{n}\right), g(y), g(y)\right)\right]=0 .
$$

Hence, it follows immediately that $g\left(u_{n}\right) \rightarrow g(x)$ and $g\left(v_{n}\right) \rightarrow g(y)$. Similarly, one can prove that $g\left(u_{n}\right) \rightarrow g\left(x^{*}\right)$ and $g\left(v_{n}\right) \rightarrow g\left(y^{*}\right)$. By uniqueness of limit, it follows that $g(x)=g\left(x^{*}\right)$ and $g(y)=g\left(y^{*}\right)$. Thus we proved (20).

Now, since $F(x, y)=g(x), F(y, x)=g(y)$ and the pair $(F, g)$ is commuting, it follows that

$$
\left\{\begin{array}{l}
g(g(x))=g(F(x, y))=F(g(x), g(y)), \\
g(g(y))=g(F(y, x))=F(g(y), g(x)) .
\end{array}\right.
$$


Denote $g(x)=z$ and $g(y)=w$. Then by (22), we deduce

$$
g(z)=F(z, w) \text { and } g(w)=F(w, z) .
$$

Thus $(z, w)$ is a coupled coincidence point. Then by (20) with $x^{*}=z$ and $y^{*}=w$, it follows that $g(z)=g(x)$ and $g(w)=g(y)$, that is $g(z)=z$ and $g(w)=w$. It follows $z=g(z)=F(z, w)$ and $w=g(w)=F(w, z)$. Therefore $(z, w)$ is a coupled common fixed point of $F$ and $g$. To prove the uniqueness, assume that $(p, q)$ is another coupled common fixed point. Then by (20), we have $p=g(p)=g(z)=z$ and $q=g(q)=g(w)=w$.

If $g=I$, the identity mapping in Theorem 2.3, then we deduce the following corollary.

Corollary 2.5. In addition to the hypotheses of Corollary 2.1, suppose that for every $(x, y),\left(x^{*}, y^{*}\right) \in X \times X$ there exists a $(u, v) \in X \times X$ such that $(F(u, v), F(v, u))$ is comparable to $(F(x, y), F(y, x))$ and $\left(F\left(x^{*}, y^{*}\right), F\left(y^{*}, x^{*}\right)\right)$. Then $F$ has a unique coupled fixed point, that is, there exists a unique $(x, y) \in X \times X$ such that $x=F(x, y)$ and $y=F(y, x)$.

Now, we give a simple illustrative example.

Example 2.1. Let $X=[0,+\infty)$. Then $(X, \preceq)$ is a partially ordered set with the partial ordering given by

$$
x \preceq y \quad \Longleftrightarrow \quad(x=y \text { or } x, y \in[0,1] \text { and } x \leq y) .
$$

Let $G(x, y, z)=|x-y|+|y-z|+|z-x|$ for $x, y, z \in X$. Define $g: X \rightarrow X$ by $g(x)=x$ for all $x \in X$ and $F: X \times X \rightarrow X$ by

$$
F(x, y)= \begin{cases}\frac{x}{8} & \text { if } x \in[0,1], y \in X \\ x-\frac{7}{8} & \text { if } x>1, y \in X .\end{cases}
$$

Define also $\phi:[0,+\infty) \rightarrow[0,+\infty)$ by $\phi(t)=\frac{t}{2}$ for all $t \in[0,+\infty)$ and $\theta:[0,+\infty) \times[0,+\infty) \rightarrow[0,+\infty)$ by $\theta\left(t_{1}, t_{2}\right)=\frac{t_{1}+t_{2}}{12}$ for all $t_{1}, t_{2} \in[0,+\infty)$. By routine calculations, the reader can easily verify that the following assumptions hold:

(i) $(X, G)$ is a complete $G$-metric space;

(ii) $F$ has the mixed monotone property;

(iii) $\left(x_{0}, y_{0}\right)=(0,1) \Rightarrow x_{0}=0=F\left(x_{0}, y_{0}\right)$ and $y_{0}=1>\frac{1}{8}=F\left(y_{0}, x_{0}\right)$;

(iv) $F$ is continuous.

Here, we show only that condition (19) in Corollary 2.1 holds for all $(x, y),(u, v),(w, z) \in$ $X \times X$ with $w \preceq u \preceq x$ and $y \preceq v \preceq z$.

We distinguish the following four cases: 
Case 1. If $(x, y),(u, v),(w, z) \in[0,1] \times[0,1]$, we have

$$
\begin{aligned}
& \phi(G(F(x, y), F(u, v), F(w, z)) \\
&= \frac{1}{2} \frac{|x-u|+|u-w|+|w-x|}{8} \\
& \leq \frac{1}{6}(|x-u|+|u-w|+|w-x|+|y-v|+|v-z|+|z-y|) \\
&= \frac{1}{4}(|x-u|+|u-w|+|w-x|+|y-v|+|v-z|+|z-y|) \\
& \quad-\frac{1}{12}(|x-u|+|u-w|+|w-x|+|y-v|+|v-z|+|z-y|) \\
&= \frac{1}{2} \phi(G(x, u, w)+G(y, v, z))-\theta(G(x, u, w), G(y, v, z)) .
\end{aligned}
$$

Case 2. If $(x, y),(u, v),(w, z) \in[0,1] \times(1,+\infty)$, we have $y=v=z$ and

$$
\begin{aligned}
& \phi(G(F(x, y), F(u, v), F(w, z)) \\
= & \frac{1}{16}(|x-u|+|u-w|+|w-x|) \\
\leq & \frac{1}{6}(|x-u|+|u-w|+|w-x|) \\
= & \frac{|x-u|+|u-w|+|w-x|}{4}-\frac{|x-u|+|u-w|+|w-x|}{12} \\
= & \frac{1}{2} \phi(G(x, u, w)+G(y, v, z))-\theta(G(x, u, w), G(y, v, z)) .
\end{aligned}
$$

Case 3. If $(x, y),(u, v),(w, z) \in(1,+\infty) \times(1,+\infty)$, we have $x=u=w$, $y=v=z$ and hence

$$
\phi(G(F(x, y), F(u, v), F(w, z))=0 .
$$

Case 4. If $(x, y),(u, v),(w, z) \in(1,+\infty) \times[0,1]$, we have again

$$
\phi(G(F(x, y), F(u, v), F(w, z))=0 .
$$

Thus condition (19) holds in all the cases. Hence by Corollary 2.1, $F$ has a coupled fixed point $(0,0) \in X \times X$.

Note that Corollary 2.1 is not applicable in respect of the usual order of real numbers because condition (19) does not hold. In fact, in this case, from condition $(19)$ with $(x, y)=(4,2),(u, v)=(3,3),(w, z)=(2,4)$, we have

$$
\begin{aligned}
& \phi(G(F(x, y), F(u, v), F(w, z))=2 \\
\not & \frac{4}{3}=\frac{1}{2} \phi(G(x, u, w)+G(y, v, z))-\theta(G(x, u, w), G(y, v, z)) .
\end{aligned}
$$

\section{REFERENCES}

[1] M. Abbas and B.E. Rhoades, Common fixed points results for non-commuting mappings without continuity in generalized metric spaces, Appl. Math. Comput. 215 (2009), 262-269. 
[2] H. Aydi, B. Samet and C. Vetro, Coupled fixed point results in cone metric spaces for $\tilde{w}$-compatible mappings, Fixed Point Theory Appl. 2011 (2011), 15 pages, doi:10.1186/1687-1812-2011-27.

[3] T.G. Bhaskar and V. Lakshmikantham, Fixed point theorems in partially ordered metric spaces and applications, Nonlinear Anal. 65 (2006), 1379-1393.

[4] B.S. Choudhury and A. Kundu, A coupled coincidence point result in partially ordered metric spaces for compatible mappings, Nonlinear Anal. 73 (2010), 2524-2531.

[5] B.S. Choudhury and P. Maity, Coupled fixed point result in generalized metric spaces, Math. Comput. Modelling 54 (2011), 73-79.

[6] R. Chugh, T. Kadian, A. Rani and B.E. Rhoades, Property $P$ in G-metric spaces, Fixed Point Theory Appl. 2010 (2010), 12 pages, Article ID 401684.

[7] J. Harijni, B. Lopez and K. Sadarangani, Fixed point theorems for mixed monotone operators and applications to integral equations, Nonlinear Anal. 74 (2011), 1749-1760.

[8] V. Lakshmikantham and $\mathrm{Lj}$. Ciric, Coupled fixed point theorems for nonlinear contractions in partially ordered metric spaces, Nonlinear Anal. 70 (2009), 4341-4349.

[9] N.V. Luong and N.X. Thuan, Coupled fixed point theorems in partially ordered Gmetric spaces, Math. Comput. Modelling 55 (2012), 1601-1609.

[10] N.V. Luong and N.X. Thuan, Coupled fixed point theorems in partially ordered metric spaces and application, Nonlinear Anal. 74 (2011), 983-992.

[11] Z. Mustafa, H. Obiedat and F. Awawdeh, Some fixed point theorem for mapping on complete metric spaces, Fixed Point Theory Appl. 2008 (2008), 12 pages, Article ID 189870.

[12] Z. Mustafa and B. Sims, A new approach to generalized metric spaces, J. Nonlinear Convex Anal. 7 (2006), 289-297.

[13] Z. Mustafa and B. Sims, Fixed point theorems for contractive mappings in complete G-metric spaces, Fixed Point Theory Appl. 2009 (2009), 10 pages, Article ID 917175.

[14] Z. Mustafa, W. Shatanawi and M. Batainesh, Esistence of fixed point results in Gmetric spaces, Int. J. Math. Math. Sci. 2009 (2009), 10 pages, Article ID 283028.

[15] H.K. Nashine, B. Samet and C. Vetro, Coupled coincidence points for compatible mappings satisfying mixed monotone property, J. Nonlinear Sci. Appl. 5 (2012), 104114.

[16] H.K. Nashine, B. Samet and C. Vetro, Monotone generalized nonlinear contractions and fixed point theorems in ordered metric spaces, Math. Comput. Modelling 54 (2011), $712-720$.

[17] R. Sadati, S.M. Vaezpour, P. Vetro and B.E. Rhoades, Fixed point theorems in generalized partially ordered G-metric spaces, Math. Comput. Modelling 52 (2010), 797-801.

[18] B. Samet, Coupled fixed point theorems for a generalized Meir-Keeler contraction in partially ordered metric spaces, Nonlinear Anal. 72 (2010), 4508-4517.

[19] B. Samet and C. Vetro, Coupled fixed point, F-invariant set and fixed point of $N$ order, Ann. Funct. Anal. 1 (2) (2010), 46-56. 
[20] B. Samet and C. Vetro, Coupled fixed point theorems for multi-valued nonlinear contraction mappings in partially ordered metric spaces, Nonlinear Anal. 74 (2011), 42604268 .

[21] W. Shatanawi, Fixed point theory for contractive mappings satisfying $\Phi$-maps in $G$ metric spaces, Fixed Point Theory Appl. 2010 (2010), 9 pages, Article ID 181650.

\section{MANish JaIN}

Department of Mathematics

Ahir College

REWARI 123401

INDIA

E-mail address: manish_261283@yahoo.com

\section{Calogero Vetro}

Università degli Studi di Palermo

Dipartimento di Matematica e Informatica

Via Archirafi 34

90123 PALERMO

ITALY

E-mail address: cvetro@math.unipa.it

Neetu Gupta

HAS DEPARTMENT

YMCAUST

FARIDABAD

INDIA

E-mail address: neetuymca@yahoo.co.in

Sanjay Kumar

Department of Mathematics

DCRUST

Murthal, Sonepat

INDIA

E-mail address: sanjaymudgal2004@yahoo.com 